\title{
Spinal Canal Stenosis
}

Spinal stenosis is defined generally as a narrowing of one or more of the following: the central canal, foramina, and lateral recesses of the lumbar spine. Specifically, this stenosis can be classified into three types as defined by location: (a) stenosis of the central spinal canal, (b) stenosis of the intervertebral spinal neural foramen or foramina, and (c) stenosis of the lateral recess(es) of the central spinal canal.

\section{CONVENTIONAL AND FAST SPIN ECHO SEQUENCES}

Although there is some clinical debate as to whether computed tomography (CT) or MR is superior in diagnosing stenosis of the spinal canal, most MR-imaging physicians feel that $T_{2}$-weighted, fat-suppressed transverse and sagittal fast spin echo imaging is the better method (Garfin et al., 1992; Hasegawa et al., 1993; Jinkins, 1999; Kent et al., 1992; Schnebel et al., 1989). Nevertheless, some clinicians still prefer conventional myelography. At present, only using conventional myelography can complete obstruction to water-soluble contrast medium flow be demonstrated, which is sometimes present in severe central spinal canal stenosis. In the future, perhaps intrathecal gadolinium-enhanced MRI will replace more conventional X-ray techniques, when and if the FDA approves this route of administration.

The following sequences comprise the preferred protocol for high-field MR machines. On some machines alternate gradient recalled echo acquisitions may be more desirable. In addition, occasionally intravenous (i.v.) contrast enhanced alternate protocols may be useful to evaluate the integrity of the blood-nerve barrier in certain cases of suspected compressive radiculopathy, or clinically ambiguous claudication possibly of spinal origin.

Table A8.2.1 lists the hardware necessary to perform the procedure, along with appropriate parameters. The available gradient strength will depend on the scanner, and the echo times (i.e., $T_{\mathrm{E}}$ ) given below in other tables will be varied accordingly (the smaller the gradient strength, the longer the echo time for a particular scan).

This entire protocol should take 35 to 45 min to complete.

NOTE: Be sure that technicians and nurses have immediate access to any emergency equipment that may be relevant to a given study, or that may be needed for a particular patient, such as crash carts or oxygen.

\section{Set up patient and equipment}

1. Interview (screen) the patient to ensure that he or she has no contraindications such as cardiac pacemakers or other implants containing ferromagnetic materials. Also be sure to find out if the patient has any health conditions that may require the presence of special emergency equipment during the scanning procedure, or necessitate any other precautions.

Table A8.2.1 Equipment Parameters for Spine Imaging in Cases of Spinal Canal Stenosis

\begin{tabular}{ll}
\hline Coil type & $\begin{array}{l}\text { Cervical, thoracic, lumbar: phase array } \\
\text { surface coil (or other depending upon } \\
\text { machine compatibility and availability) }\end{array}$ \\
Peripheral gating & Thoracic spine only (optional) \\
Respiratory gating & Thoracic spine only (optional) \\
Flow compensation pulse & Any level (optional) \\
\hline
\end{tabular}

Contributed by J. Randy Jinkins and David D. Stark 
Generally, standard screening forms are used for all patients scanned in a magnetic resonance system.

The presence of any ferromagnetic materials may be a health hazard to the patient when he or she is inside the magnet, and will also affect the imaging. If in doubt as to the exact composition of the items, it is best to exclude patients with any implants; see Shellock (1996) for discussion of what implants may be safely scanned using magnetic resonance.

Patients may be accompanied into the magnet room by a friend or family member, who can sit in the room during the scan and comfort the patient as needed. This companion must be screened as well to ensure the absence of loose metal objects on the body or clothing.

2. If the procedure is a research protocol, have the patient sign any necessary consent form.

3. Have the patient remove all jewelry and change into a gown to eliminate any metal that might be found in clothing.

4. Inform the patient about what will occur during the procedure, what he or she will experience while in the magnet, and how to behave, including the following:

a. If earphones or headphones are used to protect the ears from the loud sounds produced by the gradients, the patient will be asked to wear these, but will be able to communicate with you at any time during the imaging.

b. The patient will be given a safety squeeze-bulb or similar equipment to request assistance at any time (demonstrate how this works).

c. For good results, the patient should not talk, and should avoid or minimize swallowing or other movement, during each scan-i.e., as long as the banging sounds continue. Between scans, talking and swallowing are allowed in most cases, but should be avoided when comparative positional studies are being performed; the patient will be informed when this is the case.

d. Nevertheless, the patient may call out at any time if he or she feels it necessary.

Table A8.2.2 Primary Clinical Imaging Parameters for Sequence 1 (Pilot Scan)

Patient position
Scan type
Imaging plane (orientation)
Central slice or volume center

Echo time $\left(T_{\mathrm{E}}\right)$

Repeat time $\left(T_{\mathrm{R}}\right)$

Flip angle (FA)

Fields of view $\left(\mathrm{FOV}_{\mathrm{x}}, \mathrm{FOV}_{\mathrm{y}}\right)$

Resolution $(\Delta x, \Delta y)$

Number of data points collected $\left(N_{\mathrm{x}}, N_{\mathrm{y}}\right)$

Display matrix $\left(D_{\mathrm{x}}, D_{\mathrm{y}}\right)$

Slice thickness $(\Delta z)$

Slice gap

Number of acquisitions $\left(N_{\text {acq }}\right)$

Scan time
Supine

Gradient echo

Transverse

Laser light centered at cervical spine: thyroid cartilage; thoracic spine: nipple line; lumbar spine: iliac crests

As short as possible

As short as possible

$15^{\circ}$

Cervical: $240 \mathrm{~mm}, 240 \mathrm{~mm}$

Thoracic: $320 \mathrm{~mm}, 320 \mathrm{~mm}$

Lumbosacral: $280 \mathrm{~mm}, 280 \mathrm{~mm}$

Cervical: $0.94 \mathrm{~mm}, 0.94 \mathrm{~mm}$

Thoracic: $1.25 \mathrm{~mm}, 1.25 \mathrm{~mm}$

Lumbosacral: $1.09 \mathrm{~mm}, 1.09 \mathrm{~mm}$

256, 256

256, 256

$5 \mathrm{~mm}$

Not applicable

1

$\sim 10 \mathrm{sec}$ 
5. Have the patient mount onto the table. Either before or right after the patient lies down, set up any triggering devices or other monitoring equipment that is to be used.

6. Center the coil over the region where the key information is desired.

Make sure that the body is constrained to prevent motion, especially if high-resolution scans are to be run.

7. If needed, place a pillow or other support under the knees to make the patient more comfortable.

8. Use the centering light to position the patient and put him or her into the center of the magnet (cervical spine: thyroid cartilage; thoracic spine: nipple line; lumbar spine: iliac crests).

Table A8.2.3 Primary Clinical Imaging Parameters for Sequence 2 ( $T_{1}$-Weighted Image)

\begin{tabular}{|c|c|}
\hline Patient position & Supine \\
\hline Scan type & Conventional spin echo \\
\hline Imaging plane (orientation) & Sagittal \\
\hline \multirow[t]{4}{*}{ Central slice or volume center } & Centered on: \\
\hline & Cervical: the third cervical vertebra \\
\hline & Thoracic: the sixth thoracic vertebra \\
\hline & Lumbar: the third lumbar vertebra \\
\hline Echo time $\left(T_{\mathrm{E}}\right)$ & $10 \mathrm{msec}$ \\
\hline Repeat time $\left(T_{\mathrm{R}}\right)$ & $500 \mathrm{msec}$ \\
\hline Flip angle (FA) & $90^{\circ}$ \\
\hline \multirow[t]{3}{*}{ Fields of view $\left(\mathrm{FOV}_{\mathrm{x}}, \mathrm{FOV}_{\mathrm{y}}\right)$} & Cervical: $240 \mathrm{~mm}, 240 \mathrm{~mm}$ \\
\hline & Thoracic: $320 \mathrm{~mm}, 320 \mathrm{~mm}$ \\
\hline & $\begin{array}{l}\text { Lumbosacral: } 280 \mathrm{~mm}, 280 \mathrm{~mm} \\
\text { (may use rectangular field of view, } \\
\text { e.g., half or three-quarter field if } \\
\text { available, or tailor to region of } \\
\text { interest) }\end{array}$ \\
\hline \multirow[t]{3}{*}{ Resolution $(\Delta x, \Delta y)$} & Cervical: $0.94 \mathrm{~mm}, 0.94 \mathrm{~mm}$ \\
\hline & Thoracic: $1.25 \mathrm{~mm}, 1.25 \mathrm{~mm}$ \\
\hline & Lumbosacral: $1.09 \mathrm{~mm}, 1.09 \mathrm{~mm}$ \\
\hline Number of data points collected $\left(N_{\mathrm{x}}, N_{\mathrm{y}}\right)$ & 256,256 \\
\hline Display matrix $\left(D_{\mathrm{x}}, D_{\mathrm{y}}\right)$ & 256,256 \\
\hline \multirow[t]{3}{*}{ Slice thickness $(\Delta z)$} & Cervical: $3 \mathrm{~mm}$ \\
\hline & Thoracic: $3 \mathrm{~mm}$ \\
\hline & Lumbar: $1 \mathrm{~mm}$ \\
\hline Number of slices & $\begin{array}{l}10 \text {, or as many as needed to cover the } \\
\text { region of interest }\end{array}$ \\
\hline \multirow[t]{3}{*}{ Slice gap } & Cervical: $0.5 \mathrm{~mm}$ \\
\hline & Thoracic: $1 \mathrm{~mm}$ \\
\hline & Lumbar: $1 \mathrm{~mm}$ \\
\hline Number of acquisitions $\left(N_{\text {acq }}\right)$ & 2 \\
\hline Flow compensation & Yes (if available) \\
\hline Saturation pulses & $\begin{array}{l}\text { Yes; anterior } \\
\text { cervical/thoracic/lumbar slab to } \\
\text { saturate larynx/vessels }\end{array}$ \\
\hline Slice series & $\begin{array}{l}\text { Left to right or the reverse depending } \\
\text { on preference }\end{array}$ \\
\hline Scan time & $\sim 4 \mathrm{~min}$ \\
\hline
\end{tabular}


Table A8.2.4 Primary Clinical Imaging Parameters for Sequence 3 ( $T_{2}$-Weighted Image, $\mathrm{FSE}^{a}$ )

\begin{tabular}{|c|c|}
\hline Patient position & Supine \\
\hline Scan type & Fast spin echo \\
\hline Imaging plane (orientation) & Sagittal \\
\hline Central slice or volume center & $\begin{array}{l}\text { Centered on area of interest (as in } \\
\text { sequence 2, Table A8.2.3) }\end{array}$ \\
\hline Echo time $\left(T_{\mathrm{E}}\right)$ & $100 \mathrm{msec}$ \\
\hline Echo train length (ETL) & 8 \\
\hline Repeat time $\left(T_{\mathrm{R}}\right)$ & $4000 \mathrm{msec}$ \\
\hline Flip angle (FA) & $90^{\circ}$ \\
\hline Fields of view $\left(\mathrm{FOV}_{\mathrm{x}}, \mathrm{FOV}_{\mathrm{y}}\right)$ & As in sequence 2 (Table A8.2.3) \\
\hline \multirow[t]{3}{*}{ Resolution $(\Delta x, \Delta y)$} & Cervical: $0.47 \mathrm{~mm}, 0.47 \mathrm{~mm}$ \\
\hline & Thoracic: $0.63 \mathrm{~mm}, 0.63 \mathrm{~mm}$ \\
\hline & Lumbosacral: $0.55 \mathrm{~mm}, 0.55 \mathrm{~mm}$ \\
\hline Number of data points collected $\left(N_{\mathrm{x}}, N_{\mathrm{y}}\right)$ & 512,512 \\
\hline Display matrix $\left(D_{\mathrm{x}}, D_{\mathrm{y}}\right)$ & 512,512 \\
\hline Slice thickness $(\Delta z)$ & As in sequence 2 (Table A8.2.3) \\
\hline Number of slices & Varies with spinal level \\
\hline Slice gap & As in sequence 2 (Table A8.2.3) \\
\hline Number of acquisitions $\left(N_{\mathrm{acq}}\right)$ & 1 \\
\hline Flow compensation & Yes (if available) \\
\hline Saturation pulses & $\begin{array}{l}\text { Yes; anterior } \\
\text { cervical/thoracic/lumbar slabs to } \\
\text { saturate larynx/vessels/heart }\end{array}$ \\
\hline Fat suppression & Yes \\
\hline Slice series & $\begin{array}{l}\text { Left to right or the reverse } \\
\text { depending on preference }\end{array}$ \\
\hline Scan time & $\sim 4 \min$ \\
\hline
\end{tabular}

${ }^{a}$ FSE: fast spin echo.

Once this step has been performed, so long as the patient does not move on the table, the table itself can be moved and then replaced in the same position as before without jeopardizing the positioning of one scan relative to another.

9. If the patient is unable to hold still, provide an appropriate sedative.

\section{Sequence 1: Rapid positioning pilot}

10. To validate the patient's position, run the system's pilot (or scout) scan to (sequence 1) ensure correct location of the neck in three dimensions, using the imaging sequence given in Table A8.2.2 or similar parameters.

This sequence usually consists of three orthogonal planes to allow subsequent localization. The images are often also used later to determine where to place the saturation pulses and to set up total coverage of the volume of interest.

\section{Sequence 2: Sagittal $T_{1}$-weighted conventional spin echo}

11. Set the imaging parameters as shown in Table A8.2.3.

12. Use the pilot image to locate the spine in three dimensions to ensure coverage of the region of interest (e.g., cervical, thoracic, lumbosacral spine).

13. Let the patient know you are ready and begin the scan. 
Table A8.2.5 Primary Clinical Imaging Parameters for Sequence 4 ( $T_{1}$-Weighted Image)

\begin{tabular}{|c|c|}
\hline Patient position & Supine \\
\hline Scan type & Conventional spin echo \\
\hline Imaging plane (orientation) & Transverse \\
\hline Central slice or volume center & $\begin{array}{l}\text { Centered on the area of interest } \\
\text { (as in sequence } 2 \text {, Table A8.2.3) }\end{array}$ \\
\hline Echo time $\left(T_{\mathrm{E}}\right)$ & $10 \mathrm{msec}$ \\
\hline Repeat time $\left(T_{\mathrm{R}}\right)$ & $500 \mathrm{msec}$ \\
\hline Flip angle (FA) & $90^{\circ}$ \\
\hline Fields of view $\left(\mathrm{FOV}_{\mathrm{x}}, \mathrm{FOV}_{\mathrm{y}}\right)$ & As in sequence 2 (Table A8.2.3) \\
\hline \multirow[t]{3}{*}{ Resolution $(\Delta x, \Delta y)$} & Cervical: $0.94 \mathrm{~mm}, 0.94 \mathrm{~mm}$ \\
\hline & Thoracic: $1.25 \mathrm{~mm}, 1.25 \mathrm{~mm}$ \\
\hline & Lumbosacral: $1.09 \mathrm{~mm}, 1.09 \mathrm{~mm}$ \\
\hline Number of data points collected $\left(N_{\mathrm{x}}, N_{\mathrm{y}}\right)$ & 256,256 \\
\hline Display matrix $\left(D_{\mathrm{x}}, D_{\mathrm{y}}\right)$ & 256,256 \\
\hline \multirow[t]{3}{*}{ Slice thickness $(\Delta z)$} & Cervical: $3 \mathrm{~mm}$ \\
\hline & Thoracic: $3-5 \mathrm{~mm}$ \\
\hline & Lumbar: $4 \mathrm{~mm}$ \\
\hline Number of slices & Varies with spinal level \\
\hline \multirow[t]{3}{*}{ Slice gap } & Cervical: $1 \mathrm{~mm}$ \\
\hline & Thoracic: $1-2 \mathrm{~mm}$ \\
\hline & Lumbar: $1 \mathrm{~mm}$ \\
\hline Number of acquisitions $\left(N_{\text {acq }}\right)$ & 2 \\
\hline Slice location & See Basic Protocol, step 16 \\
\hline Flow compensation & Yes (if available) \\
\hline Saturation pulses & No \\
\hline Scan time & $\sim 4 \min$ \\
\hline
\end{tabular}

\section{Sequence 3: Sagittal $T_{2}$-weighted fast spin echo}

14. Review the pilot scans and ensure that the saturation pulse is correctly placed anterior to the slab of interest.

15. Run sequence 3 according to Table A8.2.4.

\section{Sequence 4: Transverse $T_{1}$-weighted conventional spin echo}

16. Using the midline sagittal $T_{1}$-weighted image acquired in sequence 2 , set the transverse acquisition parameters as follows:

a. Cervical spine: stacked images from C1 through C7-T1.

b. Thoracic spine: stacked images through levels of interest.

c. Lumbosacral spine: 5 slices each, angled to the plane of the intervertebral disc at L3-4, L4-5, and L5-S1; one slice each, angled to the intervertebral disc at L1-2 and L2-3.

17. Supplement additional slices according to visible disease present or to clinical query.

18. Run the sequence according to Table A8.2.5.

\section{Sequence 5: Transverse $T_{2}$-weighted fast spin echo}

19. Using the midline $T_{1}$-weighted image acquired in sequence 2 , repeat the setup as in Table A8.2.6.

20. Run sequences according to Table A8.2.6. 
Table A8.2.6 Primary Clinical Imaging Parameters for Sequence 5 ( $T_{2}$-Weighted Image, $\mathrm{FSE}^{a}$ )

\begin{tabular}{|c|c|}
\hline Patient position & Supine \\
\hline Scan type & Fast spin echo \\
\hline Imaging plane (orientation) & Transverse \\
\hline Central slice or volume center & $\begin{array}{l}\text { Centered on the region of interest } \\
\text { (as in sequence 2, Table A8.2.3) }\end{array}$ \\
\hline Echo time $\left(T_{\mathrm{E}}\right)$ & $100 \mathrm{msec}$ \\
\hline Echo train length (ETL) & 8 \\
\hline Repeat time $\left(T_{\mathrm{R}}\right)$ & $4000 \mathrm{msec}$ \\
\hline Flip angle (FA) & $90^{\circ}$ \\
\hline Fields of view $\left(\mathrm{FOV}_{\mathrm{x}}, \mathrm{FOV}_{\mathrm{y}}\right)$ & As in sequence 2, Table A8.2.3 \\
\hline \multirow[t]{3}{*}{ Resolution $(\Delta x, \Delta y)$} & Cervical: $0.94 \mathrm{~mm}, 0.94 \mathrm{~mm}$ \\
\hline & Thoracic: $1.25 \mathrm{~mm}, 1.25 \mathrm{~mm}$ \\
\hline & Lumbosacral: $1.09 \mathrm{~mm}, 1.09 \mathrm{~mm}$ \\
\hline Number of data points collected $\left(N_{\mathrm{x}}, N_{\mathrm{y}}\right)$ & 256,256 \\
\hline Display matrix $\left(D_{\mathrm{x}}, D_{\mathrm{y}}\right)$ & 256,256 \\
\hline Slice thickness $(\Delta z)$ & As in sequence 4 (Table A8.2.5) \\
\hline Number of slices & Varies with spinal level \\
\hline Slice gap & As in sequence 4 (Table A8.2.5) \\
\hline Number of acquisitions $\left(N_{\text {acq }}\right)$ & 2 \\
\hline Slice location & See Basic Protocol, step 16 \\
\hline Flow compensation & Yes (if available) \\
\hline Saturation pulses & No \\
\hline Scan time & $\sim 4 \mathrm{~min}$ \\
\hline
\end{tabular}

${ }^{a}$ FSE: fast spin echo.

\section{COMMENTARY}

\section{Background Information}

The etiology of central stenosis of the lumbosacral spinal canal may be either developmental or acquired in nature, although most cases presenting in adulthood represent a combination of these factors. Pre-existent developmental circumferential narrowing of the spinal canal coupled with hypertrophic/redundant degenerative changes of the intervertebral discs, posterior spinal facet joints, and related spinal structures/tissues results in a constriction of the thecal sac and its contents. This may engender a clinical syndrome known as "neurogenic or spinal claudication." Although it can be difficult on occasion to differentiate on a clinical basis between neurogenic and vascular forms of claudication, classically the former consists of lower extremity pain that is both poorly localized/characterized and is often associated with leg numbness and/or weakness. These signs and symptoms are frequently bilateral and are usually preceded for months or years by intractable low back pain. Neurogenic claudication is typically exacerbated by walking or standing erect and is relieved somewhat by spinal flexion. A common presenting complaint is also uni- or bilateral radiating lower extremity radicular pain. Other clinical findings such as unusual or poorly defined lower extremity paresthesias, penile erections on walking, and sexual impotence are seen with lesser frequency. The cause of this clinical syndrome is believed to be the result of chronic or intermittent progressive compression of the cauda equina at the level(s) of central spinal canal stenosis.

Pathophysiologically, the fundamental neural abnormalities include direct repeated local nerve root trauma, focal nerve root ischemia, inhibition of free access of the nerve root to the nutritional effects of free-flowing CSF (cerebrospinal fluid), obstruction of axoplasmic flow within the nerve root, axon demyelination/remyelination, and extensive axon degeneration/regeneration. It has also been shown that constriction of the lumbar canal, together with repetitive motion induced by physical flexion and extension, produces a stretching of nerve roots and thereby results in dramatic neural elongation and redundancy both above 
A

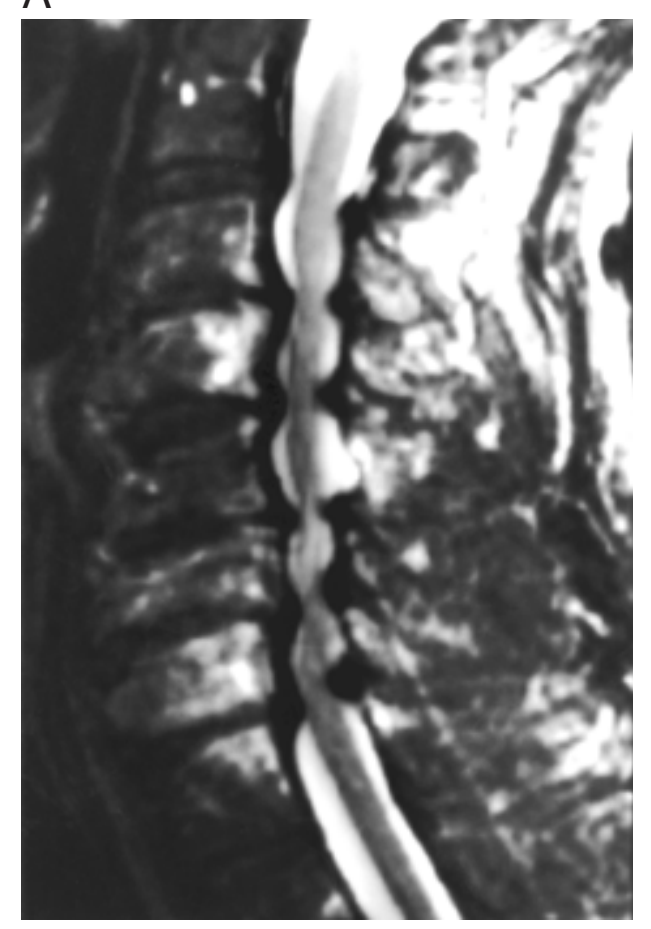

B

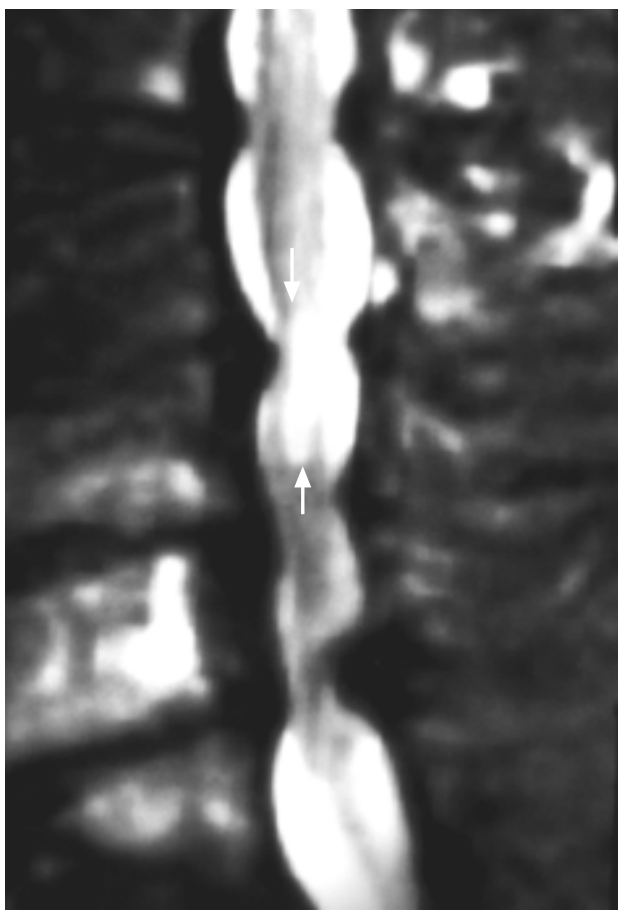

Figure A8.2.1 Cervical spinal stenosis. (A) Sagittal $T_{2}$-weighted ( $T_{\mathrm{R}}=4000 \mathrm{msec}, T_{\mathrm{E}}=100 \mathrm{msec}$ ) image shows multilevel stenosis of the central spinal canal. (B) Magnified view of (A) shows hyperintensity (arrows) within the cervical spinal cord indicating myelomalacia.

and/or below the central spinal canal stenosis. This nerve root redundancy on conventional myelographic inspection may have a serpentine configuration intradurally that simulates, and can be confused with, vascular structures (i.e., vascular malformation). It has been postulated that the major factor in the intrathecal enhancement on i.v. gadolinium-enhanced MRI seen on both sides of severe central lumbar canal stenosis represents pathologic disruption of the blood-nerve barrier within compressed, elongated, and redundant nerve roots. Among other things, it has been previously shown that benign blood-nerve barrier disruption occurs in response to mechanical trauma, Wallerian degeneration, and sterile inflammation. A contributory mechanism of intrathecal enhancement in such severe cases of central spinal stenosis may involve neural and perineural vascular engorgement and stagnation [i.e., of the great radicular $\operatorname{vein}(s)]$ that accompanies nerve root constriction, thus resulting in prominent enhancement of the vasa nervorum. From a practical standpoint, the presence of intrathecal enhancement in this setting signifies unequivocal underlying neural pathology associated with spinal disease of a stenotic nature in cases of clinical claudication of ambiguous etiology (Jinkins, 1993).

\section{Critical Parameters and Troubleshooting}

CSF flow, cardiac, laryngeal, body wall and other sources of motion can produce artifacts that may, on occasion, significantly degrade the images. Proper spatial (e.g., prevertebral) saturation pulses and sometimes cardiac/respiratory gating can reduce these artifacts significantly. In some instances, these artifacts may be difficult or impossible to easily overcome from patient to patient.

\section{Anticipated Results}

The goal of studying the spine in cases of clinically suspected spinal stenosis is to determine the level(s), region(s), and degree(s) of severity of the stenosis. The images acquired utilizing these MRI sequences will allow the most accurate assessment of the central spinal canal, lateral recesses, and spinal neural foramina that is available to the medical imaging
Extradural Spine

A8.2.7 

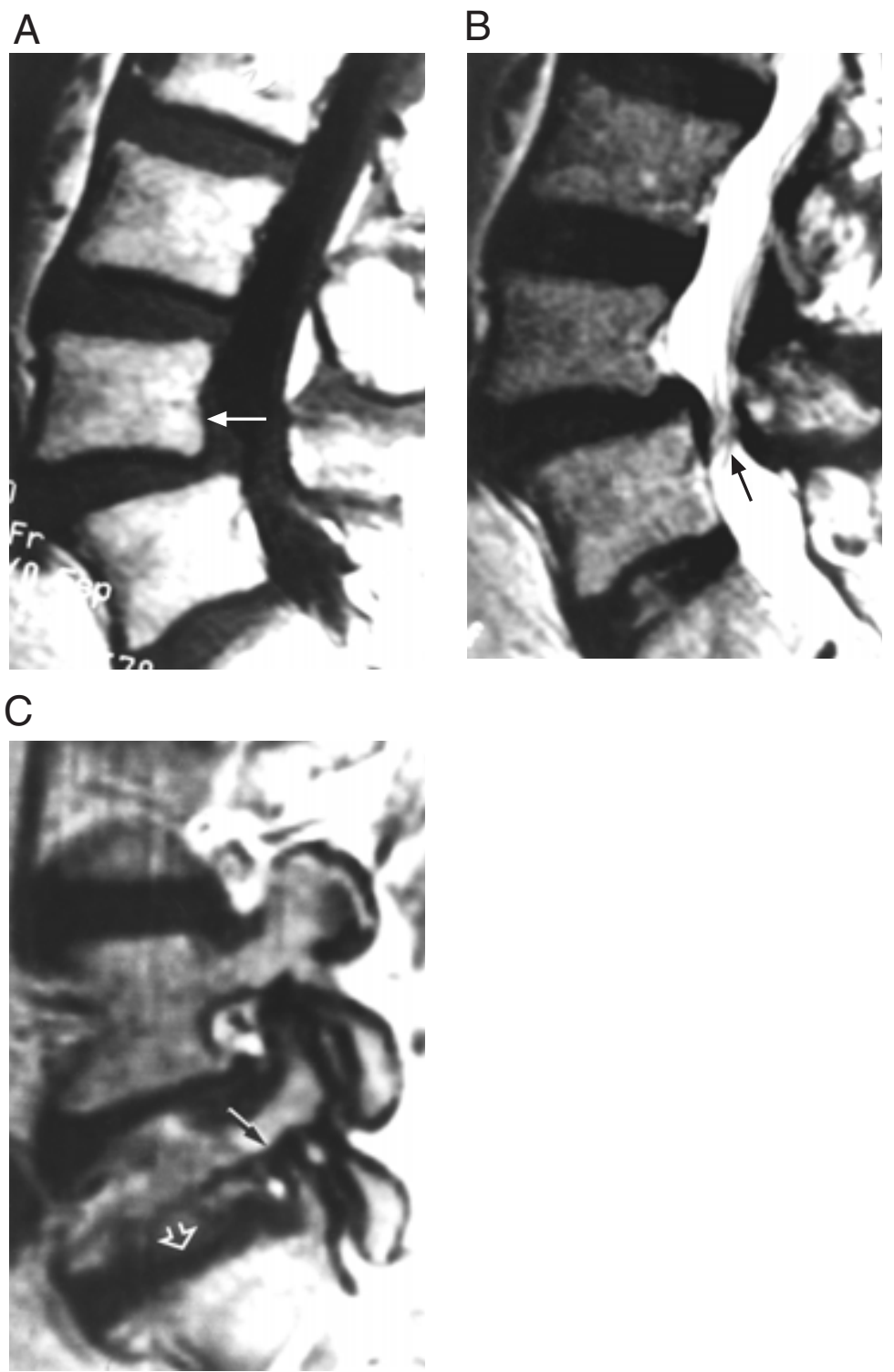

Figure A8.2.2 Lumbar spinal stenosis. (A) Sagittal $T_{1}$-weighted $\left(T_{\mathrm{R}}=500 \mathrm{msec}, T_{\mathrm{E}}=10 \mathrm{msec}\right)$ image shows degenerative anterolisthesis of $L 4$ on $L 5$ (arrow). (B) Sagittal $T_{2}$-weighted $\left(T_{R}=4000\right.$ $\mathrm{msec}, T_{\mathrm{E}}=100 \mathrm{msec}$ ) image shows stenosis of the central spinal canal (arrow). (C) Parasagittal $T_{1}$-weighted $\left(T_{\mathrm{R}}=500 \mathrm{msec}, T_{\mathrm{E}}=10 \mathrm{msec}\right.$ ) image shows partial collapse of the intervertebral disc (open arrow) and stenosis of the spinal neural foramen (solid arrow).

specialist today. Some examples are shown in Figure A8.2.1 and Figure A8.2.2.

\section{Literature Cited}

Garfin, S.R., Rydevik, B.L., Lipson, S.J., et al. 1992. Spinal stenosis. In The Spine, 3rd ed. (R.H. Rothman, F.A. Simeone, eds.) pp. 791-857. W.B. Saunders, Philadelphia.

Hasegawa, T., An, H.S., and Haughton, V.M. 1993. Imaging anatomy of the lateral lumbar spinal canal. Semin. Ultrasound CT MRI 14(6):404413.
Jinkins, J.R. 1993. Gd-DTPA enhanced MR of the lumbar spinal canal in patients with claudication. J. Assist. Comput. Tomogr. 17:555-562.

Jinkins, J.R. 1999. MR evaluation of stenosis involving the neural foramina lateral recesses and central canal of the lumbosacral spine. Neuroimag. Clin. North Am. 7:493-511.

Kent, D.L., Haynor, D.R., Larson, E.B., and Deyo, R.A. 1992. Diagnosis of lumbar spinal stenosis in adults; a metaanalysis of the accuracy of CT, MR, and myelography. Am. J. Roentgenol. 158:1135-1144. 
Schnebel, B., Kingston, S., Watkins, R., and Dillon, W. 1989. Comparison of MRI to contrast CT in the diagnosis of spinal stenosis. Spine 14:332337.

Shellock, F.G. 1996. Pocket Guide to MR Procedures and Metallic Objects. Lippincott-Raven, Philadelpia.

Contributed by J. Randy Jinkins and David D. Stark

Downstate Medical Center

State University of New York

Brooklyn, New York 